\title{
Hyperspectral Image Analysis for Precision Viticulture
}

\author{
M. Ferreiro-Armán ${ }^{1}$, J.-P. Da Costa ${ }^{2}$, S. Homayouni ${ }^{2}$ and J. Martín-Herrero ${ }^{1}$ \\ 1 Departamento de Teoría do Sinal e Comunicacións, \\ ETSET, Universidade de Vigo, E-36200, Spain \\ 2 LAPS - UMR 5131 CNRS - Université Bordeaux 1, F-33405 Talence, France *
}

\begin{abstract}
We analyze the capabilities of CASI data for the discrimination of vine varieties in hyperspectral images. To analyze the discrimination capabilities of the CASI data, principal components analysis and linear discriminant analysis methods are used. We assess the performance of various classification techniques: Multi-layer perceptrons, radial basis function neural networks, and support vector machines. We also discuss the trade-off between spatial and spectral resolutions in the framework of precision viticulture.
\end{abstract}

\section{Introduction}

Thanks to recent advances in remote sensing, there has been a growing interest in its application to precision agriculture. In the field of precision viticulture, airborne multispectral and hyperspectral imagery has made possible a flexible mapping of yield, soil properties, or diseases $[1-3,19,20]$. In less than a decade, affordable hyperspectral devices have allowed an accurate spectral characterization of the vine canopy, including variety mapping. Even if several studies have proved the relevance of spectral information for the discrimination of plant species (e.g. [4-6]), few authors have investigated the capability of remote sensed hyperspectral data for grape variety mapping $[7,8]$. At the vineyard level, there is a need for grape variety discrimination as a useful tool for vine growers to detect misplantings and to manage inner field species variability. At a regional level, varietal discrimination enables the regional mapping of vine varieties, which can be used for planning and inventory purposes. For instance, in the Bordeaux wine producing area in France, vine producer unions need tools to help in the certification of wine growers. The specifications of wine labels include limitations in the percentage of area for each grape variety as well as in the plant density. Varietal mapping is a valuable help in the certification of wine productions.

Promising results have recently been obtained for variety discrimination and misplanting detection. A recent study [8] evaluated the potential of airborne visible and near infrared hyperspectral imagery for the mapping of grape vine varieties in Southern Australia. They used twelve spectral bands obtained with a

\footnotetext{
* This work has been cofinanced with FEDER funds through the Interreg IIIb "Atlantic Area" program within the PIMHAI project.
} 
CASI (Compact Airborne Spectrographic Imager), between 490 and $801 \mathrm{~nm}$. The spatial resolution of about $1 \mathrm{~m}$ per pixel was enough to isolate the vine rows from the background. Maximum likelihood classification allowed the discrimination of two varieties: Cabernet Sauvignon and Shiraz. Good classification rates (around $90 \%$ ) were obtained by considering a vine row as a whole and by classifying the row depending on which variety was the most represented in the row.

\subsection{The CASI Sensor}

CASI is a hyperspectral sensor which collects spectral information from airborne platforms. It is a push-broom sensor based on a 512-pixel CCD. Its spectral range is [ $403 \mathrm{~nm}, 947 \mathrm{~nm}$ ], and the spatial resolution ranges from $0.6 \mathrm{~m}$ to $4 \mathrm{~m}$ with a maximum flight height around $3000 \mathrm{~m}$. Spatial resolution is controlled by flight altitude. The lower the altitude, the higher the spatial resolution. At the same flight speed, higher spatial resolution implies higher frame rate to keep pixels square, and the number of spectral bands must be reduced to fit the system bandwidth. This is the cause for the spatial/spectral resolution trade-off typical of the CASI. Additionally, the fixed lens configuration and the security limits for low flight prevent spatial resolution higher than $0.5 \mathrm{~m}$. Anyway, this spatial resolution is not of much use due to the low number of spectral bands imposed by the system bandwidth at the higher spatial resolutions.

The CASI has two main acquisition modes: the spectral mode, with a poor spatial resolution and a rich spectral information (for instance, $3 \mathrm{~m}$ per pixel and 144 spectral channels), and the spatial mode, with a high spatial resolution and a poor spectral information (for instance: $0.6 \mathrm{~m}$ per pixel, 4 spectral channels).

In the context of precision agriculture, spatial resolution is a major factor determining the accuracy in crop characterization. Precision viticulture targets vineyards, which are row crops, characterized by the alternation of vegetation and soil. The characterization of such crops by remote sensing imaging techniques requires, on one hand, a high spatial accuracy in order to separate efficiently the target from other features in the image (soil, weeds, etc.). Otherwise, spectral signatures would include significant contributions from the different non targeted elements present in the field. On the other hand, a full spectral range with narrow bands is needed for an accurate description of plant reflectance. Thus, the discrimination between vine and weeds, among vine varieties, and the detection of disease affection strongly depend on both spatial and spectral resolutions.

However, the CASI limitations stated above do not allow a high spatial resolution and a large spectral range at the same time. The spatial mode at 0.6 $\mathrm{m}$ allows only 4 spectral bands which are not enough for accurate variety discrimination at the plant level. In contrast, with 144 bands, the spectral mode provides sufficient spectral information, at the cost of very low spatial accuracy. In this work, we try to assess the discrimination capabilities of hyperspectral CASI data at the plant level on vines using the spectral acquisition mode. 


\subsection{Image Data}

The CASI data were acquired in August 2005 in the Pessac-Léognan area, in the wine growing region of Bordeaux, France. The Domain Château LucheyHalde is covered by several varieties of vine among which Cabernet Sauvignon (CS) and Merlot Noir (MN) constitute a large part of the vineyard. Four other varieties are present: Petit Verdot, Cabernet Franc, Sauvignon and Sémillon. A map of the varieties cover and the CASI image data are shown in Fig. 1. Spatial resolution is $3 \mathrm{~m}$. At this spatial resolution, the full spectral range (144 channels in $[400 \mathrm{~nm}, 950 \mathrm{~nm}]$ ) is available. Spectral resolution is $10 \mathrm{~nm}$.

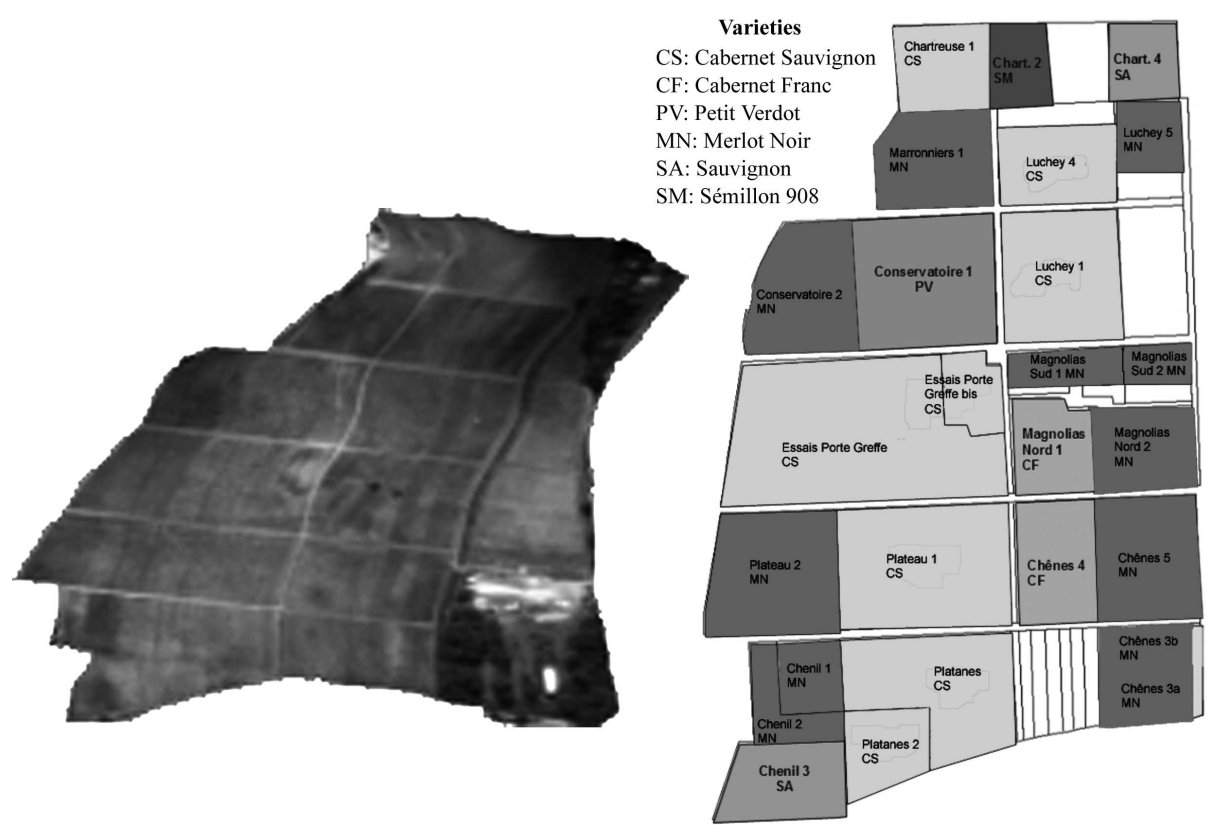

Fig. 1. Château Luchey-Halde: CASI image data (left) and varieties cover (right).

\section{Statistical methods and data sets}

\subsection{Classification methods}

The discrimination ability of CASI data is investigated using various statistical methods. A preliminary Principal component analysis (PCA) is used for an exploratory look at the data. Then, a linear discriminant analysis (LDA) $[9,10]$ is performed in a first discrimination attempt. LDA provides the discriminant function that best discriminates between varieties. Its construction is based on the first principal components only in order to avoid the classical stability problems 
linked to the high correlations usually observed on hyperspectral data [21]. This first attempt shows the capabilities of CASI data for varietal discrimination, though using a linear model only.

Finally, the discrimination ability of CASI data is investigated more thoroughly using supervised classification methods on several data sets. The classifications are performed on the whole set of 144 spectral bands using three classifiers: Multi-layer perceptrons (MLP) [11,12], radial basis function neural networks [13] initialized with a self-organizing map [14,15] (SOM-RBF), and support vector machines (SVM) [16]. We provide quantitative measures for the goodness of the classification, such as the $\kappa$ statistic [17] and the overall accuracy $(\mathrm{OA})$, as well as classification maps.

\subsection{Data Sets}

We built three data sets: One for testing the discrimination ability of the CASI data by means of PCA [18] and LDA [9,10], and two sets for classification.

Set 1 consists of 837 samples from four varieties in various parcels (Table 1).

Table 1. Data set 1.

\begin{tabular}{c|cc}
\hline Variety & \multicolumn{2}{|c}{ No. pixels Parcels } \\
\hline MN & 342 & 9 \\
CS & 394 & 8 \\
CF & 56 & 2 \\
SA & 45 & 2 \\
\hline
\end{tabular}

In sets 2 and 3, the data were split into two subsets: one subset for training and one subset for testing. Pixels within the MN and CS varieties were selected in specific parcels. See Tables 2 and 3.

Table 2. Data set 2 .

\begin{tabular}{c|cc}
\hline Class & Training & Testing \\
\hline MN & 100 & 400 \\
& Plateau 2 & Conservatoire 2, Chenil 1, 2, Marroniers, Chênes 3a, 3b, 5 \\
\hline CS & 100 & 400 \\
& Essai PG E (left) & Platanes, Plateau 1, Luchey 1, 4 \\
\hline Soil & 50 & 200 \\
\hline Trees & 50 & 200 \\
\hline
\end{tabular}


Table 3. Data set 3 .

\begin{tabular}{c|cc}
\hline Class & Training & Testing \\
\hline MN & 100 & 400 \\
& Conservatoire 2 & Plateau 2, Chenil 1, 2, Marroniers, Chênes 3a, 3b, 5 \\
\hline CS & 100 & 400 \\
& Essai PG W (right) & Platanes, Plateau 1, Luchey 1, 4 \\
\hline Soil & 50 & 200 \\
\hline Trees & 50 & 200 \\
\hline
\end{tabular}

\section{$3 \quad$ Experimental Results}

\subsection{Discrimination between Varieties CS and MN}

Principal Components Analysis on MN and CS. In this first experiment, we study whether it is possible to discriminate between two different varieties (MN and CS) using hyperspectral CASI data.

A PCA is carried out on 736 pixels of data set 1 , belonging to the MN and CS classes (342 and 394 pixels, respectively). The variables are the normalized reflectances for the 144 available spectral bands. The first four components are given in Table 4.

Table 4. PCA eigenvalues on data set 1.

\begin{tabular}{ccc}
\hline Component Variance (\%) & Cumulated \% \\
\hline 1 & 66.31 & 66.31 \\
2 & 19.05 & 85.36 \\
3 & 2.76 & 88.12 \\
4 & 0.90 & 89.02 \\
\hline
\end{tabular}

Figure 2 is a representation of the pixels in the first two factorial axes, which cumulate the majority of the information (85\%).

Clearly, the two varieties are not linearly separable. However, it seems that MN pixels lie in the bottom left part of the representation whereas CS pixels tend to the top right part. This hints at some discrimination being possible.

Linear Discriminant Analysis on MN and CS. The previous results are confirmed by a linear discriminant analysis (LDA) run on the first principal components. For this experiment, data set 1 is split in two subsets: We randomly select $20 \%$ of the pixels for the training set and the rest $(80 \%)$ for the test set. PCA on the training set leads to the following eigenvalues (Table 5).

As the first two components accumulate $88 \%$ of the total variance, only the first two principal components were kept for the LDA-based classification. The results of LDA on the training and test sets are shown in Table 6. The percentages of good classification in the training and in the test sets are $80 \%$ 


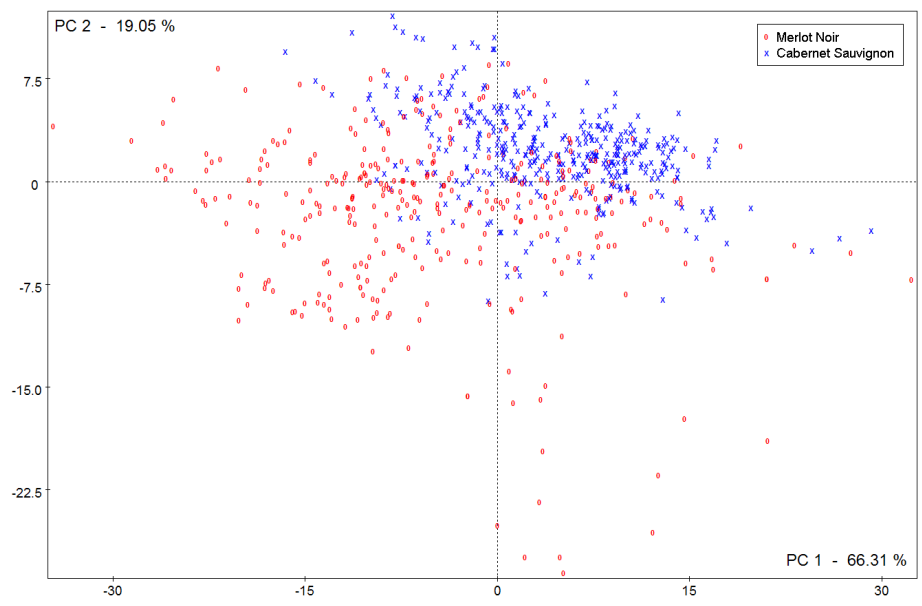

Fig. 2. Repartition of Merlot Noir and Cabernet Sauvignon varieties on the first and second PCA axes.

Table 5. PCA eigenvalues on training set 1.

\begin{tabular}{ccc}
\hline Component Variance (\%) & Cumulated \% \\
\hline 1 & 69.76 & 69.76 \\
2 & 18.24 & 88.00 \\
3 & 1.49 & 89.82 \\
4 & 1.49 & 91.31 \\
\hline
\end{tabular}

and $76 \%$ respectively, which confirms that the two varieties can be discriminated by hyperspectral data in some degree. Thus, these classification rates, obtained with linear methods, are quite promising. They are expected to improve using non-linear classification approaches.

Table 6. Results of LDA classification.

\begin{tabular}{l|cc|cc}
\multicolumn{3}{c}{ Train } & \multicolumn{2}{c}{ Test } \\
\hline$\%$ & \multicolumn{1}{c}{ MN } & CS & MN & CS \\
\hline MN & 71 & 29 & 67 & 33 \\
CS & 13 & 87 & 16 & 84 \\
\hline
\end{tabular}

To study the coherence of the results, we plotted the regression coefficients as a function of the wavelength (Fig. 3).

The regression coefficients (i.e. the weights of each wavelength in the linear discriminant function) are quite regular, allowing an interpretation, be it physical or biological, of the phenomena explaining the discrimination. However, this interpretation may remain difficult and risky since we have to keep in mind 


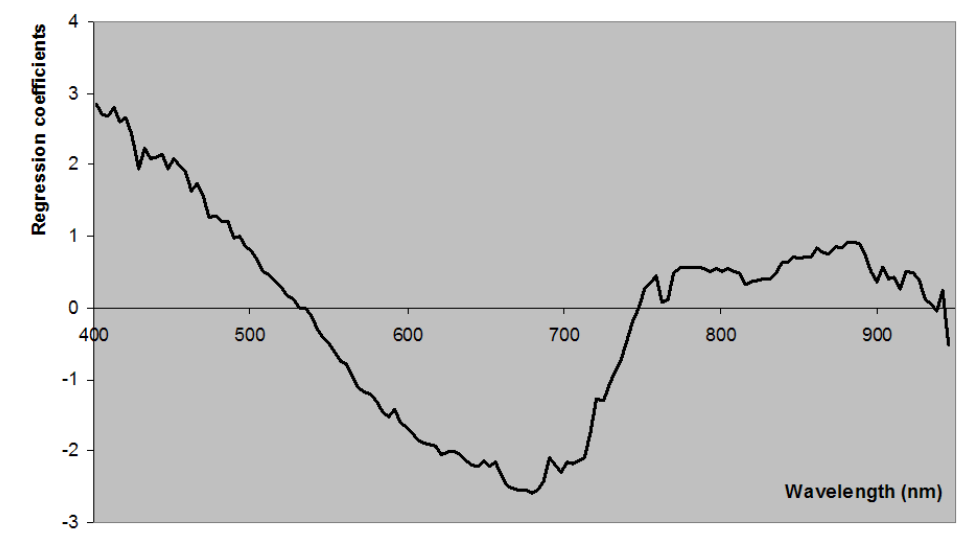

Fig. 3. Regression coefficients of the linear discriminant function between CS and MN varieties as a function of the wavelength.

that, in the CASI data we are exploring, pixels result from the mixing of several contributions, including vegetation and soil. The latter covers more than $50 \%$ of the effective surface of each pixel $\left(\sim 9 \mathrm{~m}^{2}\right)$, even inside the plots. Thus, trying to draw a causal explanation from the shape of the discriminant function may be misleading even if the discrimination is satisfactory.

Discrimination of Four Varieties. We carried out the same kind of experiment with four varieties: Merlot Noir, Cabernet Sauvignon, Cabernet Franc, and Sauvignon. A PCA was run on data set 1. The variables are the normalized reflectances for the 144 available spectral bands, see Table 7 .

Table 7. PCA on data set 1 with four varieties.

\begin{tabular}{ccc}
\hline Component Variance (\%) & Cumulated \% \\
\hline 1 & 68.00 & 68.00 \\
2 & 18.50 & 86.50 \\
3 & 3.20 & 89.60 \\
4 & 0.90 & 90.50 \\
\hline
\end{tabular}

Figure 4 represents the pixels in the first two factorial axes, which cumulate the major part of information (around 86\%). It shows some degree of discrimination between the four varieties. For instance, the Sauvignon variety lies rather on the left part, apart from the other varieties. In contrast, other varieties, like Cabernet Franc and Cabernet Sauvignon seem hardly separable. However, the reason may be physiological, as these two varieties are biologically very close.

The previous experiments have shown that, despite the low spatial resolution, the available CASI data contain sufficient spectral information to partially 


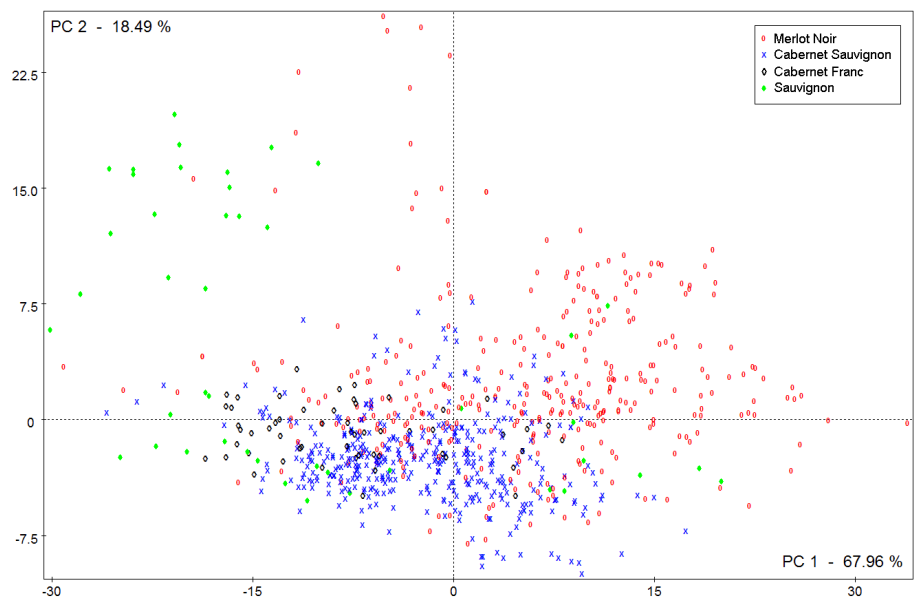

Fig. 4. Layout of the four main varieties on the first and second PCA axes.

discriminate between vine varieties. However, PCA clearly shows a high variability of spectral information within each vine variety. This variability may find explanations in the large amount of soil in each pixel and in the large diversity of soils within the domain. As we consider row crops, each pixel (which is $3 \mathrm{~m}$ wide) is a mixture between crop (vine) and non crop (soil, weeds) information. The effect of soil on each pixel reflectance can be direct or indirect. The direct effect is due to the spectral reflectance depending on the type of soil and, more particularly, on the ratio of coarse elements on the surface. The indirect effect is physiological: Depending on soil nature, the vigor of the plants may vary inside the field. As a consequence, the quantity and state of vegetation, along with the proportion of soil within each pixel, may change between and even within plots.

\subsection{Classification}

In this section, the discrimination ability of CASI data is investigated more thoroughly. Classification experiments are carried out to evaluate the influence of soil variability and plant vigor differences in species discrimination. We use supervised classification techniques described in the literature, namely multi-layer perceptron (MLP) trained with backpropagation with momentum $(\mu)$, radial basis function neural networks initialized with a self-organizing map (SOM-RBF), and Gaussian kernel support vector machines (SVM), using data sets 2 and 3 . The classifiers' configurations are displayed in Table 8.

Classification Results on Data Set 2. The composition of this data set was given in Table 2. Different plots were used for the test and train sets. Table 9 shows the $\kappa$ statistic, the overall accuracy (OA), and the confusion matrices. 
Table 8. Configuration of the classifiers used in the experiments.

\begin{tabular}{l|l}
\hline Classifier & Configuration \\
\hline MLP & $\begin{array}{l}20 \text { hidden neurons } \\
\eta=0.2, \mu=0.8,500 \text { epochs }\end{array}$ \\
\hline SOM-RBF & $\begin{array}{l}100 \text { hidden neurons } \\
\eta=0.01,200 \text { epochs }\end{array}$ \\
\hline SVM & $C=1000$ Gaussian kernel $\sigma=0.5$ \\
\hline
\end{tabular}

Table 9. Classification results with the test set for data set 2 .

\begin{tabular}{|c|c|c|c|c|c|c|c|c|c|c|c|c|c|}
\hline & \multicolumn{4}{|c|}{ MLP } & \multicolumn{4}{|c|}{ SOM-RBF } & & \multicolumn{3}{|c|}{ SVM } & \\
\hline & \multicolumn{2}{|c|}{$\kappa$} & \multicolumn{2}{|c|}{ OA } & \multicolumn{2}{|c|}{$\kappa$} & \multicolumn{2}{|c|}{ OA } & \multicolumn{2}{|c|}{$\kappa$} & \multicolumn{2}{|c|}{ OA } & \\
\hline$\%$ & \multicolumn{2}{|c|}{84.5} & \multicolumn{2}{|c|}{88.8} & 85. & & \multicolumn{2}{|c|}{89.2} & 86. & & \multicolumn{2}{|c|}{90.5} & $\%$ \\
\hline$\%$ & MN & CS & Soil & Trees & MN & $\mathrm{CS}$ & Soil & Trees & MN & $\mathrm{CS}$ & Soil & Trees & $\%$ \\
\hline$\overline{\mathrm{MN}}$ & 88 & 12 & 0 & 0 & 90 & 8 & 2 & 0 & 92 & 5 & 0 & 3 & MN \\
\hline CS & 16 & 84 & 0 & 0 & 16 & 81 & 3 & 0 & 16 & 84 & 0 & 0 & CS \\
\hline Soil & 0 & 1 & 99 & 0 & 0 & 0 & 100 & 0 & 3 & 3 & 94 & 0 & Soil \\
\hline Trees & 5 & 0 & 2 & 93 & 0 & 0 & 5 & 95 & 0 & 2 & 0 & 98 & Trees \\
\hline
\end{tabular}

All classifiers show good classification scores, with an average overall accuracy of $90 \%$. The confusion matrices show misclassifications between MN and CS pixels. Except for a few pixels, the classification of trees and soil is successful.

The classification maps are shown in Fig. 5.

As compared with the ground truth map in Fig. 1, SVM leads to a good and homogeneous classification on the parcel used for training, Plateau 2, as well as on the parcels Conservatoire 2, Marronniers, and Chenil 1 and 2, used in the test set. However, various misclassifications occur on Chênes 3 and 5 and even

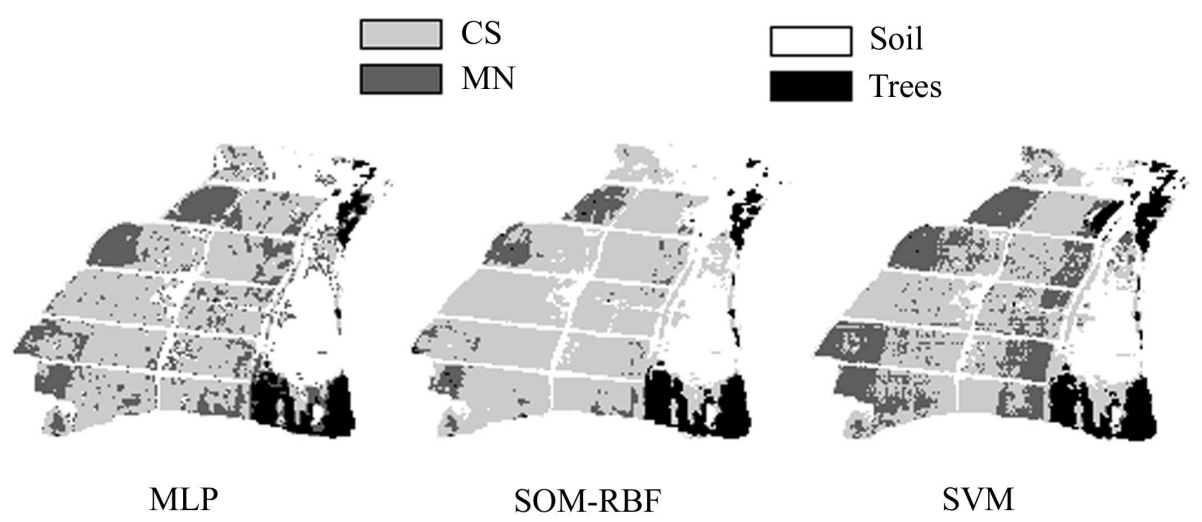

Fig. 5. Classification maps after training with set 2. 
inside the training parcel. These errors may be explained by heterogeneities in the quantity of biomass. These heterogeneities imply a strong variability in the proportions of soil and vegetation in the pixels leading to a bad influence of soil in the classification results.

Classification Results on Data Set 3. In this experiment, the classifiers are trained on the right part of the parcel Essai PG (see Table 3 for the composition of this data set), which show some parts with a weaker vegetation density, and on the parcel Conservatoire 2. Table 10 shows the $\kappa$ statistic, overall accuracy (OA), and the confusion matrices.

Table 10. Classification results with the test set for data set 3 .

\begin{tabular}{|c|c|c|c|c|c|c|c|c|c|c|c|c|c|}
\hline & \multicolumn{4}{|c|}{ MLP } & \multicolumn{4}{|c|}{ SOM-RBF } & & \multicolumn{3}{|c|}{ SVM } & \\
\hline & \multicolumn{2}{|c|}{$\kappa$} & \multicolumn{2}{|c|}{$\mathbf{O A}$} & \multicolumn{2}{|c|}{$\kappa$} & \multicolumn{2}{|c|}{$\mathbf{O A}$} & \multicolumn{2}{|c|}{$\kappa$} & \multicolumn{2}{|c|}{ OA } & \\
\hline$\%$ & \multicolumn{2}{|c|}{84.3} & \multicolumn{2}{|c|}{88.7} & & & \multicolumn{2}{|c|}{90.1} & & & \multicolumn{2}{|c|}{88.2} & $\%$ \\
\hline$\overline{\%}$ & MN & $\mathrm{CS}$ & Soi & Trees & MN & CS & Soil & Trees & MN & CS & Soil & Trees & $\%$ \\
\hline$\overline{M N}$ & 77 & 23 & 0 & 0 & 86 & 14 & 0 & 0 & 71 & 28 & 0 & 1 & MN \\
\hline CS & 7 & 93 & 0 & 0 & 9 & 91 & 0 & 0 & 3 & 96 & 1 & 0 & CS \\
\hline Soil & 0 & 4 & 96 & 0 & 0 & 4 & 96 & 0 & 0 & 1 & 99 & 0 & Soil \\
\hline Trees & 0 & 2 & 0 & 98 & 5 & 0 & 0 & 95 & 0 & 0 & 0 & 100 & Trees \\
\hline
\end{tabular}

The classification outputs are similar to those obtained with data set 2 . The classification maps in Fig. 6 show how slight differences on vegetation density do not have a strong influence over classification, but strong differences cause noticeable effects.

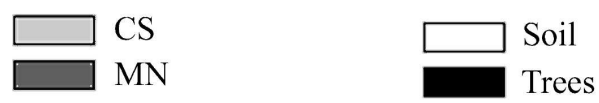

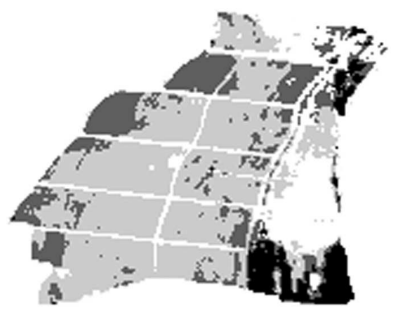

MLP

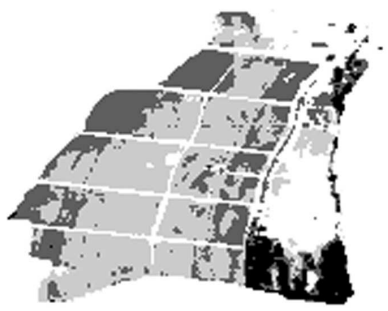

SOM-RBF

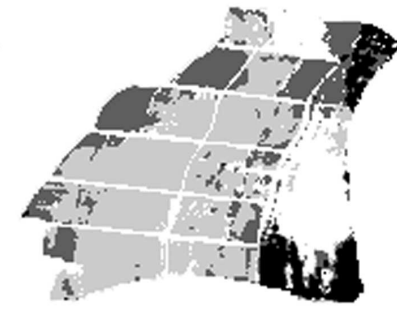

SVM

Fig. 6. Classification maps after training with set 3 . 


\section{Conclusions}

In this work, we assess the capability of an airborne hyperspectral sensor for the discrimination of vine varieties by remote sensing. With overall accuracies of about $90 \%$, classification results are promising. They suggest that such CASI data could be used for a coarse discrimination of grape varieties but cannot be trusted for an accurate (close to 100\%) discrimination.

Indeed, in the framework of precision agriculture, the CASI shows strong constraints which hinder the huge potential of imaging spectroscopy, conversely the spatial to spectral resolution trade off, which does not allow producing images with both good spatial and spectral resolution. At the higher spatial resolutions, the sensor is no longer hyperspectral, with just a few bands, even if narrow. On the other hand, at full spectral resolution, in the so called spectral mode, the low spatial resolution implies a high degree of mixture in the pixels' signatures. In the specific case of vineyards, the periodicity of vines within the parcels and the variability in the foliage induces a strong influence of the soil on the discrimination of pixels. The ratio of misclassifications suggests that these spectral mode data can not be used for an accurate pixel-wise discrimination between varieties. Indeed, even if some degree of discrimination can be reached with two classes (Merlot Noir and Cabernet Sauvignon) the results are not perfect. Moreover, taking into account several varieties does not seem feasible for the moment. For these reasons, the CASI data used in this study does not seem adequate for an accurate characterization of vegetation at the pixel scale.

Nevertheless, grape variety discrimination may be enhanced by considering higher level information on plots and parcels. At the vine row level, a solution would consist in considering the vine rows individually as it is done in [7]. Such an approach would need a row extraction step (see $[19,20]$ or $[7]$ for examples). However, the lack of spatial resolution in the spectral mode makes the CASI data hardly appropriate for such a task. Maybe the best way to deal with variety discrimination is to work at the scale of the plot. The knowledge of cadastral information about the boundings of the plots would allow variety classification at the plot level, with a higher overall accuracy than the one obtained at the pixel level. Future works will concern this aspect.

\section{Ackowledgements}

This work has been cofinanced with FEDER funds through the Interreg IIIb "Atlantic Area" program within the PIMHAI project. We are also grateful to the vine grower Château Luchey-Halde and to the ENITA de Bordeaux for allowing image acquisitions. Finally, we wish to thank the Oenologie-Ampélologie laboratory, INRA, for their valuable technical support. 


\section{References}

1. Johnson, L., Lobitz, B., Armstrong, R., Baldy, R., Weber, E., de Benedictis, J., Bosch, D.: Airborne imaging aids vineyard canopy evaluation, California Agriculture 50 (1996) 14-18.

2. Bramley, R. G. V., Proffitt, A. P.: Managing variability in viticultural production, Grapegrower and Winemaker (1999) 11-16.

3. Lamb, D. W.: Monitoring vineyard variability from the air, Australian Viticulture 3 (1999) 22-23.

4. Cochrane, M. A.: Using vegetation reflectance variability for species level classification of hyperspectral data, Int. Journal of Remote Sensing 21 (2000) 2075-2087.

5. Lewis, M. M., Jooste, V., de Gasparis, A. A.: Discimination of arid vegetation with Airborne Multispectral Scanner hyperspectral imagery, IEEE Transactions on Geoscience and Remote Sensing 39 (2001) 1471-1479.

6. Lacar, F. M., Lewis, M. M., Grierson, I. T.: Use of hyperspectral reflectance for discrimination between grape varieties, Proceedings of the International Geoscience and Remote Sensing Symposium, Sydney, Australia (2001)

7. Arkun S. A., Honey F., Johnson L., Lamb D., Lieff W., Morgan G.: Airborne remote sensing of the vine canopy. In Lamb, D., (Ed.), Vineyard Monitoring and Management beyond 2000, Charles Stuart University, Wagga Wagga (2001)

8. Lacar, F. M., Lewis, M. M., Grierson, I. T.: Use of hyperspectral imagery for mapping grape varieties in the Barossa Valley, South Australia, Proceedings of the Int. Geoscience and Remote Sensing Symposium, Sydney, Australia (2001)

9. Fisher, R. A.: The use of multiple measures in taxonomic problems, Annals of Eugenics 7 (1936) 179-188.

10. Duda, R., Hart, P., Stork, D.: Pattern Classification (Second Edition), Wiley, New York (2000)

11. McCullogh, W. S., Pitts, A.: A logical calculus of the ideas immanent in nervous activity, Bulletin of Mathematical Biophysics 5 (1943) 115-133

12. Werbos, P. J.: Beyond regression: new tools for prediction and analysis in the behavioural sciences (1974)

13. Tou, J. T., González, R. C.: Pattern Recognition, Reading, MA: Addison-Wesley (1974)

14. Kohonen, T.: Self-organizing Maps, Springer-Verlag (1997)

15. Mekkaia, Z., Mesbahi, L., Lakehal, M.: Initalizing of RBF centers by SOM algorithms, MS'2002 Int. Conf. on Modelling and Simulation in Technical and Social Sciences, Girona, Spain (2002) 713-723.

16. Vapnik, V. N.: The nature of statistical learning theory, Springer, New York (1995)

17. Foody, G. M.: On the compensation for chance agreement in image classification accuracy assessment, Photogrammetric Engineering and Remote Sensing 58 (1992) 1459-1460

18. Parkkinen, J., Jaaskelainen, T.: Color representation using statistical pattern recognition, Applied Optics 26 (1987) 4240-4245

19. Bobillet, W., Da Costa, J. P., Germain, C., Grenier, G.: Row detection in high resolution remote sensing images of vine fields, Proceedings of the 4th European Conference on Precision Agriculture (2003) 81-87

20. Michelet, F., Da Costa, J. P., Germain, C., Grenier, G.: Delineation of vine fields by segmentation of high resolution remote sensed images, Proceedings of the 5 th European Conference on Precision Agriculture (2005) 933-940.

21. Esbensen, K.H.: Multivariate data analysis in practice - an introduction to multivariate data analysis and experimental design, Camo AS (2002). 